\title{
Staphylococcus saprophyticus and Escherichia coli: Tracking from sperm fertility potential to assisted reproductive outcomes
}

\author{
Fatemeh Ghasemian, Shahin Esmaeilnezhad, Mohammad Javad Mehdipour Moghaddam \\ Department of Biology, Faculty of Sciences, University of Guilan, Rasht, Iran
}

Objective: Bacteriospermia and urogenital infections are common problems in male infertility. This study aimed to evaluate the effects of bacteriospermia on sperm parameters and clinical outcomes in semen samples infected with two common bacteria (Staphylococcus saprophyticus and Escherichia coli) in northern Iran.

Methods: Microbiological tests were performed to isolate and identify organisms from 435 semen samples from infertile couples. Semen samples were assessed according to the World Health Organization criteria. The protamine status, chromatin structure, chromatin condensation, and acrosome reaction of sperm and assisted reproductive outcomes were determined in couples with different male infertility factors.

Results: Among the total cases, the two most prevalent pathogens were considered: S. saprophyticus (38.2\%) and E. coli (52.9\%). In the semen samples infected with $E$. coli, the spontaneous acrosome reaction and abnormal chromatin condensation were more common $(p<0.05)$. Significant increases in abnormal chromatin condensation and deprotamination were seen in the presence of S. saprophyticus. In washed semen, tight adhesion between the sperm midpiece and S. saprophyticus was observed. There was also a significant decrease in the fertilization rate using semen samples infected with $S$. saprophyticus and $E$. coli during in vitro fertilization cycles $(p<0.001)$. In addition, the presence of $S$. saprophyticus and $E$. coli in semen samples was associated with a lower likelihood of clinical pregnancy in couples with various factors of male infertility.

Conclusion: Poor results of assisted reproductive techniques may be correlated with semen samples infected with two common bacteria in northern Iran.

Keywords: Assisted reproductive technique; Bacterial Infections; Fertilization; Pregnancy; Semen analysis

\section{Introduction}

Approximately $15 \%$ of cases of male infertility are due to urogenital tract inflammation and infection [1]. Clinical data show that local inflammation or infection can be seen in up to $60 \%$ of patients re-

Received: October 20, 2020 · Revised: January 18, 2021 · Accepted: February 14, 2021

Corresponding author: Fatemeh Ghasemian

Department of Biology, Faculty of Sciences, University of Guilan, Rasht 413351914 , Iran

Tel: +98-13-33343630 Fax: +98-13-33367066 E-mail: ghasemian@guilan.ac.ir

This is an Open Access article distributed under the terms of the Creative Commons Attribution Non-Commercial License (http://creativecommons.org/licenses/by-nc/4.0/) which permits unrestricted non-commercial use, distribution, and reproduction in any medium, provided the original work is properly cited. ceiving treatment with assisted reproductive technology (ART) [2]. Infections are the main origin of inflammatory disorders in the male genital tract. However, controversy exists regarding the role of different types of bacteria in decreasing sperm parameters. Therefore, male fertility may be inhibited by the negative effects of bacteriospermia and leukocytospermia on sperm parameters [2]. Existing evidence suggests a connection between bacteriospermia and alterations of semen quality. In recent years, the detrimental effects of different types of bacteria strains and/or leukocytes on some sperm parameters were studied [3]. However, the premise that the direct effects of bacteria are the reason for decreasing the chance of success in ART has not been confirmed.

Several studies have investigated the effects of different bacteria 
on semen parameters and male fertility. However, to the best of our knowledge, no study in the literature has yet investigated the impact of Staphylococcus saprophyticus on sperm quality and male fertility potential during in vitro fertilization (IVF) techniques. However, the effects of Escherichia coli have been explored in some research. Therefore, a comparative study of these two bacteria on semen parameters and fertility results of sperm in laboratory conditions would help to better understand the potential effects of bacteria on sperm fertility potential. Since the assisted reproductive outcomes of ejaculated semen with bacteriospermia are an interesting subject, we conducted this study to determine the prevalence of bacteriospermia in infertile men in northern Iran. In addition, the effects of bacteriospermia on sperm quality and assisted reproductive outcomes were studied among couples with different factors of male infertility.

\section{Methods}

\section{Semen sample collection and preparation}

After excluding infertile couples with female-factor infertility, semen samples were collected from 607 infertile couples with male factor infertility who were undergoing IVF-intracytoplasmic sperm injection (IVF-ICSI) cycles at the Alzahra Educational and Remedial Center (IVF center) from May 2017 to June 2019 (Figure 1). The exclusion criteria were men having at least one of the following conditions: (1) taking hormone-containing medications; (2) diabetes; (3); thyroid disease; (4) a history of diseases affecting the reproductive tract (e.g., cryptorchidism, varicocele, testicular torsion, chictic, or other causes of infertility that had been medically proven); (5) a history of surgery of the reproductive system, male factor infertility with previous failed IVF-ICSI cycles, and/or hormonal abnormalities; (6) heavy smoking or alcohol use; and (7) exposure to physical or chemical agents with known negative reproductive effects that may influence the semen quality. In addition, semen samples infected with leukocytes were excluded from this study. Samples infected with other bacterial species than S. saprophyticus and E. coli were excluded because the number of semen samples infected with other bacterial species was very small. Hence, statistically significant associations with clinical outcomes would not have been found (Figure 1).

The semen samples were analyzed according to the World Health Organization (WHO) criteria [4]. The semen volume, pH, sperm motility, concentration, and viability were evaluated. According to the WHO criteria [4], the semen samples were classified as showing nor-

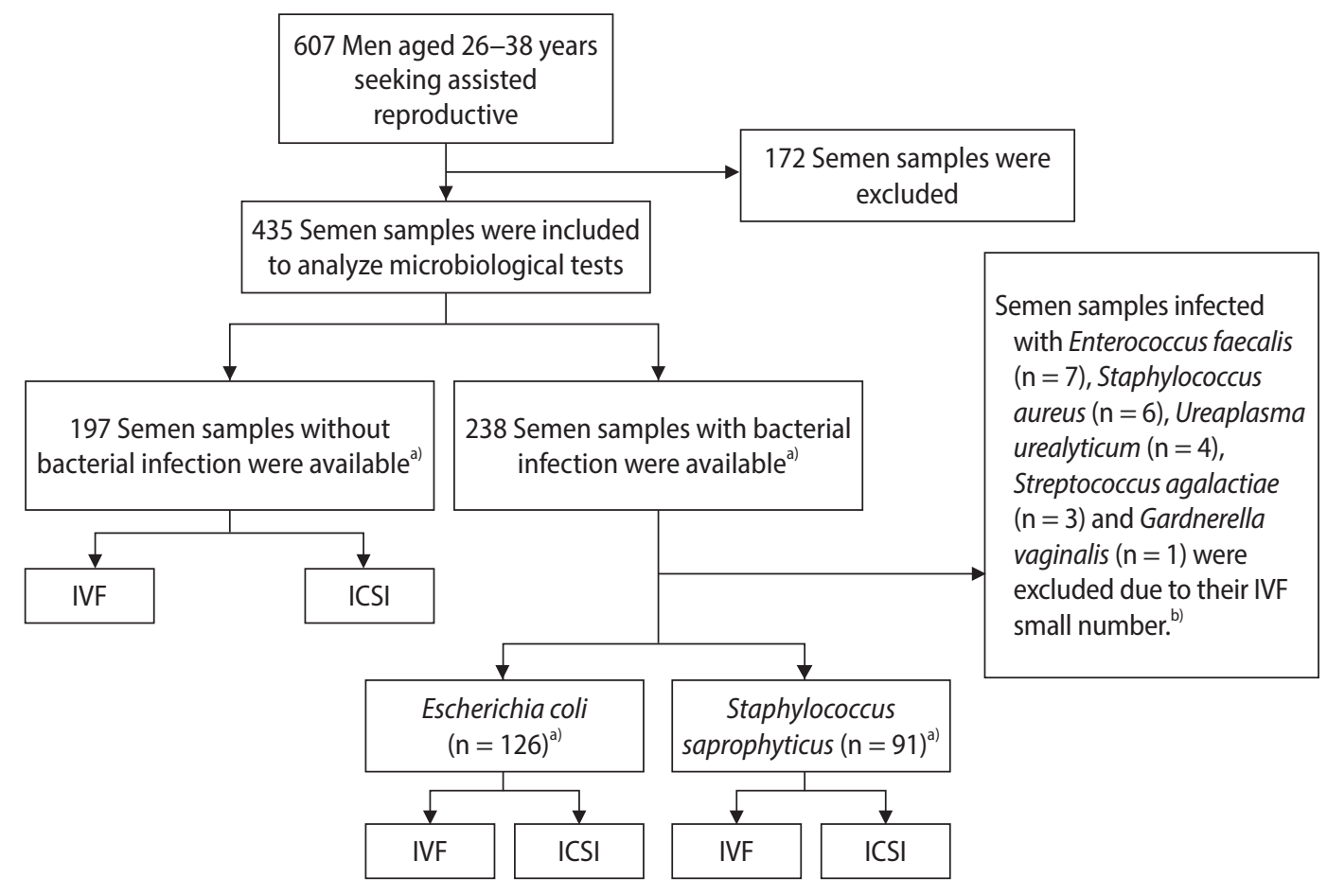

Figure 1. Flowchart of the study design. IVF, in vitro fertilization; ICSI, intracytoplasmic sperm injection. ${ }^{\text {a) }}$ All normal and infected semen samples were categorized according to World Health Organization criteria into four groups as following: normozoospermia, asthenozoospermia, teratozoospermia, and oligoasthenoteratozoospermia. ICSI and/or IVF procedures were performed for each group based

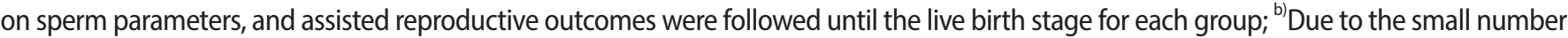
of semen samples infected with these bacteria, statistically significant associations with clinical outcomes would not have been found. 
mozoospermia, teratozoospermia, asthenozoospermia, or oligoasthenoteratozoospermia. The severity of abnormalities in the semen samples in each studied group (e.g., asthenozoospermia, teratozoospermia, and oligoasthenoteratozoospermia) was similar. This study was approved by the Guilan University of Medical Sciences committee, and informed consent was obtained from all volunteers to participate in this study.

\section{Seminal leukocyte quantification}

To distinguish white blood cells (WBCs) in semen samples, samples were studied in high-power fields. Only samples with $\geq 5$ round cells were assessed with peroxidase staining based on the Endtz test [5]. Peroxidase-positive leukocytes were indicated as stained brown, counted, and reported as $\times 10^{6} \mathrm{WBC} / \mathrm{mL}$ semen.

\section{Microbiological analysis}

Initially, patients received comprehensive information about the method of semen sample collection, the period of sexual abstinence, and the need to avoid taking antibiotics for at least 1 week before semen collection. Semen samples were collected by masturbation after 3-4 days of sexual abstinence. Patients were asked to urinate and wash their hands, penis, and scrotum before ejaculation to avoid possible contamination from the urine or external genitalia. Therefore, semen collection was done in a sterile container, following all given guidance. The semen samples were transported within 1 hour from collection to the Microbiology Laboratory of the University of Guilan to screen for a comprehensive range of microbiological organisms such as anaerobic and aerobic organisms as well as fungi and mycoplasma. First, the semen samples diluted with sterile saline (1:10) were centrifuged at $300 \times g$ for 15 minutes. The sediment was sown using $10-\mu \mathrm{L}$ calibrated loops on selective specific media. The elimination of seminal plasma and condensation of bacteria increased culture sensitivity [6].

All cultures were incubated at $37^{\circ} \mathrm{C}$ and the culture media, incubation times, and conditions were as follows: (1) blood agar to culture aerobic bacteria under aerobic conditions for 24 hours; (2) Sabouraud agar to culture fungi under aerobic conditions for 48 hours; and (3) chocolate agar to culture other microorganisms at $5 \% \mathrm{CO}_{2}$ for 48 hours. A number of isolated colonies of pathogenic bacteria higher than $1 \times 10^{3}$ colony-forming units per milliliter was considered as a positive result. Microorganisms were determined using antimicrobial sensitivity testing according to standard conventional techniques such as Gram staining, oxidase, catalase, and other biochemical tests.

\section{Sperm assays}

\section{1) Toluidine blue stain}

Toluidine blue (TB) and aniline blue (AB) staining have been introduced as sensitive tests to distinguish abnormalities in sperm chromatin structure and abnormal condensation, respectively. Thin smears were prepared on silane-coated slides. In TB staining, the sperm heads with normal chromatin structure were observed as light blue, while those with diminished integrity and abnormal chromatin structure were shown as deep violet/purple. In addition, in AB staining, at least 200 spermatozoa were counted and recorded as sperm with dark-blue (abnormal) and colorless (normal) heads. The positive group (test group; $n=6$ ) was also evaluated following the incubation of semen samples with S. saprophyticus and E. coli in vitro.

\section{2) Acrosome reaction assessment}

Triple staining was used to evaluate the acrosome reaction (acrosome integrity or reacted acrosome) according to the Talbot and Chacon method [7]. In brief, 2\% trypan blue was added to spermatozoa (1:1), and incubated at $37^{\circ} \mathrm{C}$ for 15 minutes. Fixation was done using glutaraldehyde ( $3 \%$ glutaraldehyde in $0.1 \mathrm{M}$ cacodylate buffer at $\mathrm{pH} 7.4$ ) for 30 to 60 minutes. After centrifugation (at $600 \times g$ for 5 minutes), the smear was prepared from fixed sperm and stained with Bismarck Brown $Y$ at $40^{\circ} \mathrm{C}$ for 5 minutes. The slides were then stained in Rose Bengal at $24^{\circ} \mathrm{C}$ for $20-45$ minutes. Washing and dehydration were performed in water and an alcohol series, respectively. A total of 300 spermatozoa in each slide cleared with xylene and protected with a coverslip were examined using a light microscope. The positive group (test group; $n=6$ ) was also evaluated following the incubation of semen samples with $S$. saprophyticus and $E$. coli in vitro.

\section{3) Determination of sperm chromatin packaging by chromomycin $\mathrm{A} 3$}

Chromomycin A3 (CMA3) stains guanosine-cytosine-rich sequences as a polymerase inhibitor fluorochrome, which distinguishes the degree of sperm protamination [8]. In this way, air-dried smears of spermatozoa were fixed in methanol/glacial acetic acid (3:1) for 20 minutes at $4^{\circ} \mathrm{C}$. The slides were then air-dried at room temperature and stained with $100 \mu \mathrm{L}$ of CMA3 solution $(0.25 \mathrm{mg} / \mathrm{mL}$ CMA3 in Mcllvaine's buffer, containing $10 \mu \mathrm{M} \mathrm{MgCl}$ ) (Figure 2). The positive group (test group; $n=5$ ) was also evaluated following the incubation of semen samples with S. saprophyticus and $E$. coli in vitro.

\section{4) Scanning electron microscopy}

The semen samples prepared by density gradient centrifugation were fixed with Karnovsky solution for 30 minutes at $4^{\circ} \mathrm{C}$. Centrifuga- 


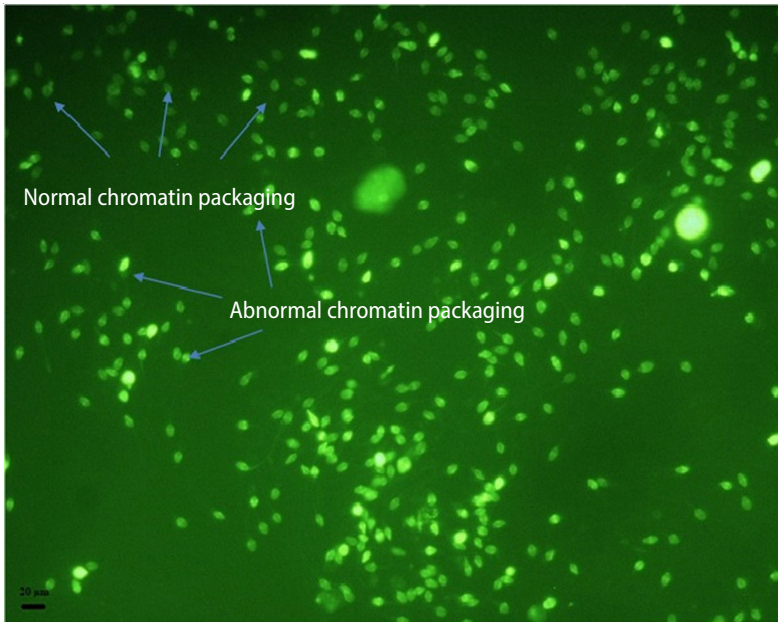

Figure 2. Sperm deprotamination. Spermatozoa with negative (dull yellow/normal) and positive (bright yellow/abnormal) chromomycin A3 staining. Scale bar $=20 \mu \mathrm{m}$.

tion was done at 4,000 $\times g$ for 15 minutes. After washing the samples, post-fixation was performed with $1 \%$ osmium tetroxide for 30 minutes. Dehydration was then done in ascending grades of ethanol, with drying at a critical point using $\mathrm{CO}_{2}$ (Balzers CPD-010). Gold-coated specimens (Balzers MED-010) were examined using a Philips FEM 515 scanning electron microscope (SEM). The positive group (test group; $n=4$ ) was also evaluated following the incubation of semen samples with S. saprophyticus and $E$. coli in vitro. The adhesion of bacteria to sperm structures was compared to the sham group (human spermatozoa incubated with S. saprophyticus and E. coli under in vitro conditions) [2].

\section{ICSI and IVF laboratory procedures}

On the day of oocyte retrieval, semen samples were collected via masturbation, liquefied, and prepared by density gradient centrifugation (90 and 45\% SpermGrade; Vitrolife, Sweden, Göteborg). Semen parameters were assessed according to the WHO [4] criteria. The ICSI or IVF procedure was performed up to 3-4 hours after sperm preparation. The selection of sperm for IVF or ICSI was based on sperm morphology, motility, concentration, patient's history, infertility etiology, and female age. The evaluation of fertilization was performed 16-18 hours after ICSI/IVF with the observation of the 2-pronuclear stage. The embryo development rate was also evaluated at 2-3 days. Intrauterine embryo transfer (ET) was performed at 2-3 days after microinjection. Increasing serum beta-human chorionic gonadotropin concentrations and detection of the fetal heartbeat were defined as biochemical and clinical pregnancy at 2 and 4 weeks after ET, respectively.

\section{Statistical analysis}

The prevalence of bacteriospermia was calculated from the proportion of positive cases relative to the total number of the study population and expressed as a percentage. Using linear regression, the relationship between bacteriospermia and other semen parameters was analyzed. The Fisher exact test was performed to assess the fertilization and cleavage rate, clinical pregnancy rate, and live birth rate. The statistical analysis was done using IBM SPSS ver. 20 (IBM Corp., Armonk, NY, USA). The $P$-values $<0.05$ were considered to indicate statistical significance.

\section{Results}

\section{Semen samples}

In this study, the women and men had a mean age of $26.0 \pm 3.2$ years (range, $22-32$ years) and $32.0 \pm 5.9$ years (range, $26-38$ years), respectively. Statistically significant differences were not found for the age $(p>0.05)$ or body mass index (BMl; $p>0.05)$ of the female partners in the normozoospermia. In addition, these similar results were seen about groups of asthenozoospermia, teratozoospermia, and oligoasthenoteratozoospermia ( $p>0.05$ ). Men's age and BMI also did not show significant differences in different groups $(p>0.05)$. Therefore, positive or negative effects of these parameters on ART outcomes were excluded.

Microbiological analyses were done for semen samples from 435 infertile men, of which 197 semen cultures were negative (45.2\%) and 238 (54.7\%) were positive. E. coli and S. saprophyticus were the most commonly isolated pathogens $(52.9 \%$ and $38.2 \%$, respectively). Semen samples infected with Enterococcus faecalis ( $n=7,2.9 \%)$, Staphylococcus aureus $(n=6,2.5 \%)$, Ureaplasma urealyticum $(n=4$, $1.6 \%)$, Streptococcus agalactiae ( $n=3,1.2 \%)$, and Gardnerella vaginalis $(n=1,0.4 \%)$ were also detected. Those bacterial species other than S. saprophyticus and E. coli were excluded to trace reproductive outcomes due to the small number of semen samples, as a result of which statistical significance would not be expected for associations with clinical outcomes. The infection severity of semen samples with S. saprophyticus and E. coli was almost identical. Mixed infections with both bacteria (S. saprophyticus and E. coli) were not observed in any of the cultured semen samples. Therefore, the quality of semen samples infected with each type of bacteria (S. saprophyticus or $E$. coli) and their clinical outcomes were analyzed.

Among the 414 specimens analyzed based on the WHO criteria [4], 141 (34.05\%) had normozoospermia, 100 (24.15\%) had teratozoospermia, 103 (24.87\%) had asthenozoospermia, and 70 (19.9\%) had oligoasthenoteratozoospermia. 


\section{Sperm parameters}

As seen in Table 1, the percentage of sperm deprotamination (seen by CMA3 staining) in normozoospermia samples infected with $S$. saprophyticus $(258 / 950,27.15 \%)$ was significantly higher $(p<0.05)$ than that of the non-bacteriospermia samples (116/825, 14.06\%). This rate was higher, at $37.8 \%(p<0.01)$ and $36.8 \%(p<0.05)$, in the asthenozoospermia and teratozoospermia samples infected with $S$. saprophyticus, respectively. The evaluation of acrosome integrity showed that the rate of premature acrosome reaction was higher in the oligoasthenoteratozoospermia samples infected with $E$. coli $(279 / 856,33.5 \%$; $p<0.05)$. Sperm viability significantly declined in all samples infected with $E$. coli $(p<0.05)$. A decrease of viability was also seen in the asthenozoospermia $(p<0.001)$ and teratozoospermia $(p<0.05)$ samples infected with S. saprophyticus $(54.9 \%$ and $59.02 \%$, respectively). In addition, the evaluation of abnormalities in sperm chromatin condensation using the $A B$ stain showed that the rate of abnormal sperm chromatin condensation (dark sperm) was higher in both bacteriospermia groups in the samples with different infertility factors (Table 1).

\section{SEM images of bacteriospermia}

SEM evaluation was performed for each sample prepared by density gradient centrifugation. The tight adhesion between bacteria and spermatozoa was found. Adhesion of S. saprophyticus to the tail of the sperm, especially to the sperm midpiece, was observed. However, the frequency of these adhesions was significantly higher in the asthenozoospermia and teratozoospermia samples from patients with male factor infertility. The adhesion of both bacteria types to spermatozoa was found in the unwashed semen samples; however, after washing, adhesion of S. saprophyticus was still observed, but less frequently (Figure 3).

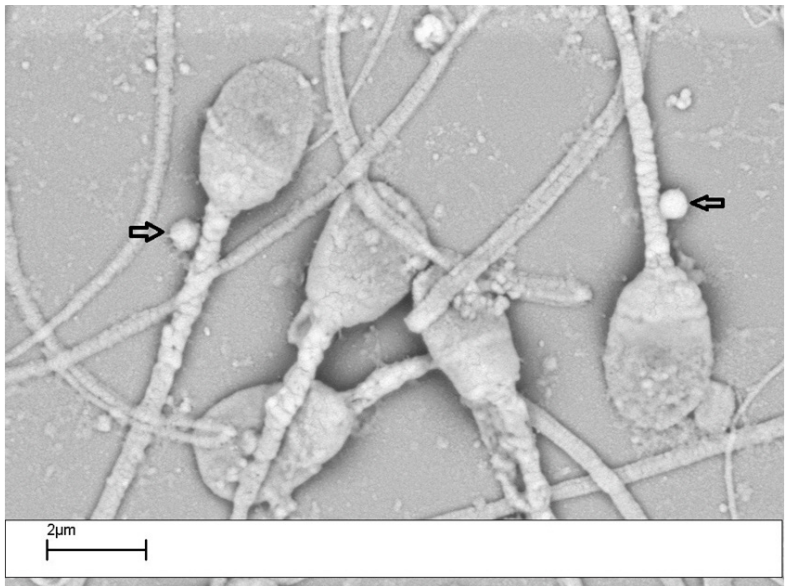

Figure 3. Interaction between bacteria and human spermatozoa. Scanning electron micrographs of prepared human spermatozoa after density gradient centrifugation. Adhesion of Staphylococcus saprophyticus to the sperm midpiece (arrows) was observed.

Table 1. The effect of bacteriospermia on semen quality in men with various infertility factors

\begin{tabular}{|c|c|c|c|c|c|c|}
\hline Variable & No. of patients & CMA3 (\%) & Spontaneous AR (\%) & $A B(\%)$ & TB (\%) & Viability (\%) \\
\hline \multicolumn{7}{|l|}{ Normozoospermia } \\
\hline Staphylococcus saprophyticus & 41 & $27.2 \pm 3.2^{\mathrm{a})}$ & $20.1 \pm 2.2$ & $22.6 \pm 2.8$ & $25.7 \pm 2.6$ & $68.6 \pm 10.8$ \\
\hline Escherichia coli & 36 & $20.1 \pm 2.2$ & $26.2 \pm 2.9$ & $29.7 \pm 2.4^{\mathrm{a})}$ & $27.8 \pm 2.4$ & $64.1 \pm 9.4^{b)}$ \\
\hline Nonbacteriospermia & 64 & $14.1 \pm 2.6$ & $18.1 \pm 1.9$ & $16.8 \pm 1.8$ & $20.1 \pm 2.9$ & $82.2 \pm 10.9$ \\
\hline \multicolumn{7}{|l|}{ Asthenozoospermia } \\
\hline Staphylococcus saprophyticus & 19 & $37.8 \pm 2.5^{b)}$ & $26.4 \pm 2.2$ & $31.6 \pm 2.7$ & $25.8 \pm 2.6$ & $54.9 \pm 9.2^{c)}$ \\
\hline Escherichia coli & 30 & $23.8 \pm 2.2$ & $29.9 \pm 2.3$ & $29.1 \pm 2.1$ & $26.9 \pm 2.4$ & $53.4 \pm 9.1^{c)}$ \\
\hline Nonbacteriospermia & 54 & $19.1 \pm 1.9$ & $19.2 \pm 2.4$ & $21.8 \pm 2.2$ & $22.4 \pm 2.6$ & $79.5 \pm 10.0$ \\
\hline \multicolumn{7}{|l|}{ Teratozoospermia } \\
\hline Staphylococcus saprophyticus & 15 & $36.8 \pm 3.6^{a)}$ & $28.6 \pm 2.9$ & $36.4 \pm 3.3^{\mathrm{a})}$ & $30.4 \pm 3.8$ & $59.0 \pm 9.5^{\mathrm{a})}$ \\
\hline Escherichia coli & 35 & $26.6 \pm 2.4$ & $31.8 \pm 3.3$ & $34.7 \pm 3.6$ & $33.6 \pm 3.4$ & $53.1 \pm 9.1^{b)}$ \\
\hline Nonbacteriospermia & 50 & $22.3 \pm 3.2$ & $24.3 \pm 2.4$ & $23.6 \pm 2.8$ & $26.4 \pm 2.6$ & $70.8 \pm 8.3$ \\
\hline \multicolumn{7}{|l|}{ Oligoasthenoteratozoospermia } \\
\hline Staphylococcus saprophyticus & 16 & $39.3 \pm 3.4$ & $27.4 \pm 2.2$ & $38.7 \pm 3.5^{\mathrm{a})}$ & $35.7 \pm 3.6$ & $53.4 \pm 9.4$ \\
\hline Escherichia coli & 25 & $32.4 \pm 3.1$ & $33.5 \pm 2.7^{\mathrm{a})}$ & $37.4 \pm 2.7^{\mathrm{a})}$ & $37.4 \pm 2.5$ & $51.9 \pm 8.3^{\mathrm{a})}$ \\
\hline Nonbacteriospermia & 29 & $28.6 \pm 2.6$ & $20.9 \pm 2.2$ & $21.6 \pm 2.3$ & $30.1 \pm 2.6$ & $61.6 \pm 9.1$ \\
\hline
\end{tabular}

Values are presented as mean \pm standard deviation. The semen samples infected with S. saprophyticus showed significant differences in terms of sperm quality such as sperm deprotamination (normozoospermia, asthenozoospermia, and teratozoospermia), abnormalities in sperm chromatin condensation (teratozoospermia and oligoasthenoteratozoospermia), and viability (asthenozoospermia and teratozoospermia) in comparison to the control group (non-bacteriospermia). In addition, the semen samples infected with $E$. coli showed significant differences in sperm quality such as abnormal chromatin condensation (normozoospermia and oligoasthenoteratozoospermia), spontaneous acrosome reaction (oligoasthenoteratozoospermia), and viability (normozoospermia, asthenozoospermia, teratozoospermia, and oligoasthenoteratozoospermia) in comparison to the control group (non-bacteriospermia). $C M A 3$, chromomycin $A 3 ; A R$, acrosome reaction; $A B$, aniline blue; $T B$, toluidine blue.

${ }^{\text {a) }} p<0.05 ;{ }^{\text {b) }} p<0.01 ;{ }^{c} p<0.001$. 


\section{ICSI and IVF outcomes}

Table 2 shows the clinical outcomes of IVF and ICSI cycles. The clinical results revealed that there was no significant difference in the fertilization rate of ICSI cycles from normozoospermia samples. However, the fertilization rate of normozoospermia samples infected with S. saprophyticus $(p<0.001)$ and E. coli $(p<0.05)$ was lower in the IVF cycles. No significant difference was found in the embryo development rate of groups (normozoospermia, asthenozoospermia, and oligoasthenoteratozoospermia, $p>0.05$ ), except for the teratozoospermia samples infected with $S$. saprophyticus $(p<0.05)$. In contrast, the clinical pregnancy rate was lower for the semen samples infected with $E$. coli from ICSI/IVF cycles in each group. Therefore, in the normozoospermia group, the clinical pregnancy rate decreased in samples infected with $E$. coli during ICSI cycles $(p<0.001)$ and in both bacteriospermia groups during IVF cycles $(p<0.001)$. Bacteriospermia significantly influenced the clinical pregnancy rate in the asthenozoospermia (S. saprophyticus and E. coli, $p<0.001$ ) and teratozoospermia (E. coli, $p<0.05$ ) groups (Table 2).

\section{Discussion}

The present study investigated the effects of the most common bacteria (S. saprophyticus and $E$. coli) on sperm parameters and assisted reproductive outcomes in infertile couples with male factor infertility in northern Iran. Teratozoospermia was the most common abnormality in semen samples infected with S. saprophyticus, as manifested by sperm deprotamination, sperm chromatin condensation, and impaired viability. Other semen samples were also influenced by infection with S. saprophyticus and E. coli. Therefore, semen samples infected with S. saprophyticus showed significant differences in terms of sperm quality such as sperm deprotamination (normozoospermia and asthenozoospermia), abnormalities in sperm chromatin condensation (oligoasthenoteratozoospermia), and impaired viability (asthenozoospermia). In addition, semen samples infected with E. coli showed significant differences in terms of sperm quality such as abnormal chromatin condensation (normozoospermia and oligoasthenoteratozoospermia), spontaneous acrosome reaction (oligoasthenoteratozoospermia), and impaired viability (normozoospermia, asthenozoospermia, teratozoospermia, and oligoasthenoteratozo-

Table 2. The effect of bacteriospermia on assisted reproductive outcomes in men with different infertility factors

\begin{tabular}{|c|c|c|c|c|c|}
\hline Variable & & Fertilization rate (\%) & Cleavage rate (\%) & Clinical pregnancy rate P/ET (\%) & Live birth LB/IE (\%) \\
\hline \multicolumn{6}{|l|}{ Normozoospermia } \\
\hline \multirow[t]{2}{*}{ Staphylococcus saprophyticus } & ICSI & 72.2 & 83.4 & $8 / 14(57.1)$ & $4 / 8(50.0)$ \\
\hline & IVF & $56.2^{\mathrm{b})}$ & 78.1 & $8 / 20(40.0)^{b)}$ & $4 / 8(50.0)$ \\
\hline \multirow[t]{2}{*}{ Escherichia coli } & ICSI & 67.2 & 85.9 & $8 / 17(47.1)^{b)}$ & $3 / 8(37.5)^{a)}$ \\
\hline & IVF & $67.3^{\mathrm{a})}$ & 81.6 & $6 / 13(46.2)^{b)}$ & $3 / 6(50.0)$ \\
\hline \multirow[t]{2}{*}{ Nonbacteriospermia } & ICSI & 74.0 & 83.5 & $17 / 26(65.4)$ & $10 / 17(58.8)$ \\
\hline & IVF & 79.6 & 85.5 & $22 / 30(73.3)$ & $13 / 22(59.1)$ \\
\hline \multicolumn{6}{|l|}{ Asthenozoospermia } \\
\hline Staphylococcus saprophyticus & ICSI & 68.4 & 82.1 & $7 / 15(46.7)^{b)}$ & $3 / 7(42.8)$ \\
\hline Escherichia coli & ICSI & 69.1 & 83.4 & $12 / 25(48.0)^{b)}$ & $4 / 12(33.3)^{a)}$ \\
\hline Nonbacteriospermia & ICSI & 72.7 & 89.3 & $33 / 49(67.3)$ & $18 / 33(54.5)$ \\
\hline \multicolumn{6}{|l|}{ Teratozoospermia } \\
\hline Staphylococcus saprophyticus & ICSI & 55.9 & $72.8^{\mathrm{a})}$ & $6 / 13(46.2)$ & $2 / 6(33.3)^{a)}$ \\
\hline Escherichia coli & ICSI & 57.1 & 78.2 & $9 / 28(32.1)^{a)}$ & $3 / 9(33.3)^{a)}$ \\
\hline Nonbacteriospermia & ICSI & 59.1 & 85.0 & 19/41 (46.3) & $10 / 19(52.6)$ \\
\hline \multicolumn{6}{|l|}{ Oligoasthenoteratozoospermia } \\
\hline Staphylococcus saprophyticus & ICSI & 55.7 & 74.1 & $5 / 14(35.7)$ & $1 / 5(20.0)^{\mathrm{a})}$ \\
\hline Escherichia coli & ICSI & 60.3 & 79.8 & $8 / 21(38.1)$ & $2 / 8(25.0)$ \\
\hline Nonbacteriospermia & ICSI & 58.1 & 76.2 & $10 / 26(38.5)$ & $3 / 10(30.0)$ \\
\hline
\end{tabular}

Values are presented as number (\%) unless otherwise indicated. The semen samples infected with S. saprophyticus showed significant differences in terms of assisted reproductive outcomes such as the fertilization rate (normozoospermia), embryo cleavage rate (teratozoospermia), clinical pregnancy rate (normozoospermia and asthenozoospermia), and live birth rate (oligozoospermia and oligoasthenoteratozoospermia) in comparison to the control group (non-bacteriospermia). In addition, the semen samples infected with E. coli showed significant differences in the fertilization rate (normozoospermia), pregnancy rate (normozoospermia, asthenozoospermia, and teratozoospermia), and live birth rate (asthenozoospermia and teratozoospermia) in comparison to the control group (non-bacteriospermia).

$P$, positive cycle; ET, embryo transfer; LB, live birth; IE, implanted embryo; ICSI, intracytoplasmic sperm injection; IVF, in vitro fertilization.

a) $p<0.05 ;{ }^{\text {b) }} p<0.001$. 
ospermia). The poor sperm quality in the semen samples infected with bacteria may have affected the assisted reproductive outcomes. Furthermore, tight adhesion of S. saprophyticus to sperm after density gradient centrifugation was shown, which is reflected in the IVF outcomes (fertilization and clinical pregnancy rates). Although many pathogenic bacteria have been recognized as potential causes of infertility, the resultant clinical outcomes in IVF/ICSI procedures have not yet been elucidated [9]. This study showed that S. saprophyticus can interact with spermatozoa, causing reduced acrosomal function, abnormal chromatin condensation (AB staining), sperm deprotamination (CMA3 staining), and tight adhesion to different parts of the tail. Reduced motility was seen in this group. These changes were reflected in IVF outcomes, as the fertilization rate of IVF cycles was lower than that of ICSI cycles for the normozoospermia samples.

The relationship between bacteriospermia and fertilization potential remains unclear [10]. The impact of genital tract infections and bacterial contamination of semen on male fertility potential has been examined in many studies; nevertheless, the impact of bacteriospermia on fertility potential has yet to be elucidated, and there is not complete agreement on the detrimental effect of bacterial infections on sperm quality $[10,11]$. The most frequently isolated microorganisms in semen have been reported to be U. urealyticum, E. coli, E. faecalis, and S. faecalis [10], but few studies have investigated the role of S. saprophyticus in semen quality and male fertility potential. Therefore, the clinical significance of the presence of bacteria in the seminal fluid is still a matter of debate [12]. The present study demonstrates that $E$. coli and S. saprophyticus reduce sperm fertility potential and impair assisted reproductive outcomes.

Some researchers have reported that no semen parameters were significantly influenced in specimens with bacteriospermia [13-17]. In addition, Sanocka-Maciejewska et al. [18] observed no effect of bacteria isolated from the genitourinary tract on semen parameters in normozoospermia samples; nevertheless, a reduced antioxidant capacity of sperm was found in infertile patients. In contrast, other studies have reported that bacteriospermia was significantly associated with semen parameters such as sperm count, motility, and morphology [19]. Attachment between E. coli and sperm, morphological abnormalities, and sperm agglutination have been reported in some studies [20]. A direct negative effect of certain pathogens on the function of sperm and oocyte quality has been proven [21]. In some works, it has also been reported that sperm parameters such as sperm count, motility (both in vitro and in vivo), morphology, and viability decreased in the presence of $E$. coli $[3,18,19]$, which is in line with our results. As the results of this study show, the quality and function of sperm decreased in the presence of bacteria, such as a declined protamination rate (positive CMA3 staining), a higher rate of premature acrosome reaction, reduced viability, and abnormal chromatin condensation ( $A B$ staining). This negative correlation between sperm quality and bacterial infection may be due to increased reactive oxygen species (ROS) production associated with inflammatory processes in semen infections, as some studies demonstrated that enhanced ROS production led to impairment of sperm DNA and male fertility [9].

In the present study, a diminished quality of sperm infected with $S$. saprophyticus and E. coli was followed by a decrease in the clinical pregnancy rate during ICSI/IVF cycles. It can be inferred that bacterial infections of the semen affect the fertility potential of men, even in ART under in vitro conditions. Therefore, the existence of bacteria and interactions between bacteria and sperm may explain why the fertilization rate and clinical pregnancy rate were lower in the semen samples infected with some bacteria.

In conclusion, bacterial infections of the male urogenital tract have been correlated with subfertility and infertility. However, these effects on fertility potential are multifunctional and complex. One relevant factor is microbial infection of the semen. This study showed negative correlations between two prevalent bacteria in the north of Iran (E. coli and S. saprophyticus) and sperm quality and assisted reproductive outcomes such as reduced viability, premature acrosome reaction, a lower protamination rate using CMA3 staining, abnormal chromatin condensation using $A B$ staining, adhesion to sperm, change of sperm function, and lower fertilization and clinical pregnancy rates. To the best of our knowledge, no studies have previously evaluated the presence and effect of these bacteria on sperm quality and fertility potential during ICSI/IVF cycles. Therefore, the results of this study can be useful for promoting assisted reproductive outcomes.

\section{Conflict of interest}

No potential conflict of interest relevant to this article was reported.

\section{Acknowledgments}

The authors of this study acknowledge the cooperation of the co-workers at the IVF center of Alzahra Hospital (Rasht, Iran) and at the Guilan Microbiology Laboratory (Rasht, Iran).

\section{ORCID}

Fatemeh Ghasemian ～https://orcid.org/0000-0001-9284-6545

Shahin Esmaeilnezhad https://orcid.org/0000-0001-6279-6209

Mohammad Javad Mehdipour Moghaddam

https://orcid.org/0000-0002-0392-065X 


\section{Author contributions}

Conceptualization: FG. Data curation: SE, MJMM. Formal analysis: FG. Methodology: SE. Project administration: FG, MJMM. Visualization \& Writing-original draft: FG. Writing-review \& editing: FG, MJMM.

\section{References}

1. Pellati D, Mylonakis I, Bertoloni G, Fiore C, Andrisani A, Ambrosini G, et al. Genital tract infections and infertility. Eur J Obstet Gynecol Reprod Biol 2008;140:3-11.

2. Fraczek M, Kurpisz M. Mechanisms of the harmful effects of bacterial semen infection on ejaculated human spermatozoa: potential inflammatory markers in semen. Folia Histochem Cytobiol 2015;53:201-17.

3. Fraczek M, Wiland E, Piasecka M, Boksa M, Gaczarzewicz D, Szumala-Kakol A, et al. Fertilizing potential of ejaculated human spermatozoa during in vitro semen bacterial infection. Fertil Steril 2014;102:711-9.e1

4. World Health Organization. WHO laboratory manual for the examination and processing of human semen. 5th ed. Geneva: World Health Organization; 2010.

5. Shekarriz M, Sharma RK, Thomas AJ Jr, Agarwal A. Positive myeloperoxidase staining (Endtz test) as an indicator of excessive reactive oxygen species formation in semen. J Assist Reprod Genet $1995 ; 12: 70-4$.

6. Vicari E, Mongioi A, Speciale A, Caccamo F, Calogero A, Gulizia S, et al. Enhancing detection of gonococcus in ejaculates of adult males using sperm dilution. Arch Androl 1986;16:19-23.

7. Talbot P, Chacon RS. A triple-stain technique for evaluating normal acrosome reactions of human sperm. J Exp Zool 1981;215: 201-8.

8. Franco JG Jr, Mauri AL, Petersen CG, Massaro FC, Silva LF, Felipe V, et al. Large nuclear vacuoles are indicative of abnormal chromatin packaging in human spermatozoa. Int J Androl 2012;35:46-51.

9. Jedrzejczak P, Fraczek M, Szumala-Kakol A, Taszarek-Hauke G, Pawelczyk L, Kurpisz M. Consequences of semen inflammation and lipid peroxidation on fertilization capacity of spermatozoa in in vitro conditions. Int J Androl 2005;28:275-83.
10. Fraczek M, Hryhorowicz M, Gill K, Zarzycka M, Gaczarzewicz D, Jedrzejczak P, et al. The effect of bacteriospermia and leukocytospermia on conventional and nonconventional semen parameters in healthy young normozoospermic males. J Reprod Immunol 2016;118:18-27.

11. Golshani M, Taheri S, Eslami G, Suleimani Rahbar AA, Fallah F, Goudarzi H. Genital tract infection in asymptomatic infertile men and its effect on semen quality. Iran J Public Health 2006;35:81-4.

12. Ombelet W, Bosmans E, Janssen M, Cox A, Vlasselaer J, Gyselaers W, et al. Semen parameters in a fertile versus subfertile population: a need for change in the interpretation of semen testing. Hum Reprod 1997;12:987-93.

13. McGowan MP, Burger HG, Baker HW, de Kretser DM, Kovacs G. The incidence of non-specific infection in the semen in fertile and sub-fertile males. Int J Androl 1981;4:657-62.

14. Berger RE, Karp LE, Williamson RA, Koehler J, Moore DE, Holmes KK. The relationship of pyospermia and seminal fluid bacteriology to sperm function as reflected in the sperm penetration assay. Fertil Steril 1982;37:557-64.

15. Huwe P, Diemer T, Ludwig M, Liu J, Schiefer HG, Weidner W. Influence of different uropathogenic microorganisms on human sperm motility parameters in an in vitro experiment. Andrologia 1998;30 Suppl 1:55-9.

16. Cottell E, Harrison RF, McCaffrey M, Walsh T, Mallon E, Barry-Kinsella C. Are seminal fluid microorganisms of significance or merely contaminants? Fertil Steril 2000;74:465-70.

17. Domes T, Lo KC, Grober ED, Mullen JB, Mazzulli T, Jarvi K. The incidence and effect of bacteriospermia and elevated seminal leukocytes on semen parameters. Fertil Steril 2012;97:1050-5.

18. Sanocka-Maciejewska D, Ciupinska M, Kurpisz M. Bacterial infection and semen quality. J Reprod Immunol 2005;67:51-6.

19. Isaiah IN, Nche BT, Nwagu IG, Nnanna II. Current studies on bacterospermia the leading cause of male infertility: a protege and potential threat towards mans extinction. N Am J Med Sci 2011; 3:562-4.

20. Kaur K, Prabha V. Spermagglutinating Escherichia coli and its role in infertility: in vivo study. Microb Pathog 2014;69-70:33-8.

21. Diemer T, Ludwig M, Huwe P, Hales DB, Weidner W. Influence of urogenital infection on sperm function. Curr Opin Urol 2000;10: 39-44. 